\title{
COVID-19 and Retinal Toxicity of Anti-Malarials
}

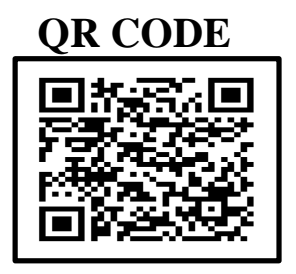

\section{MOHIT BHARTI}

The use of chloroquine and hydroxychloroquine in the tough time of COVID-19 has gained much attention. The production and use of these drugs has drastically increased in the past few months. However, it is important to know that retinal toxicity is a very rare side effect of these therapies that can lead to permanent vision loss. The mechanism is however unclear, but this toxicity might depend on the tolerance of the subjects exposed. It is important to choose the appropriate dosage which should be customized as per the patient's condition, age, body weight and comorbidities. Also, to avoid the occurrence of retinopathy, eye examination and ophthalmic screening for the patients being administered these drugs should be done. This review discusses the risk determinants, the clinical presentations and screening techniques of retinal toxicity.

KEYWORDS: Retinal Toxicity, Retinopathy, Coronavirus, Chloroquine, Hydroxychloroquine

\section{INTRODUCTION}

The exact in vitro inhibitory mechanism of Chloroquine on corona virus has already been published in a study report. ${ }^{1}$ Based on relevant studies on chloroquine and hydroxychloroquine in the treatment of COVID-19, the inclusion of these drugs was recommended for treating this pandemic. Both chloroquine and its analogue hydroxychloroquine fall into the 4aminoquinoline class of antimalarials and have some application in the treatment of rheumatoid arthritis, systemic lupus erythematosus, and other connective tissue diseases in addition to malaria. Its adverse effects include gastrointestinal reactions, skin and hair damage, neuromuscular abnormalities and eye lesions. ${ }^{2}$

Coronavirus is a kind of enveloped positive-sense RNA virus named after its coronal appearance under electron microscope. SARS-CoV-2, severe acute respiratory syndrome coronavirus and Middle East respiratory syndrome coronavirus belong to $\beta$ genus coronavirus. ${ }^{3}$ Early studies of SARS-CoV found that chloroquine could inhibit coronavirus replication by reducing terminal glycosylation of ACE2 receptors on the cell surface and interfering with viral binding to angiotensin converting enzyme 2 receptors. ${ }^{4}$ Because the SARS-CoV-2 gene sequence has high homology with SARS-CoV5, especially its similarity to the spike glycoprotein bound by the cell surface ACE2 receptor $^{6}$, the researchers used chloroquine to treat SARS-CoV-2 infected Vero E6 cells according to the mechanism and confirmed that it had a clear inhibitory effect on SARS-CoV-2 in vitro. ${ }^{1}$

In ophthalmic clinical work, the retinal toxicity of CQ and HCQ is a common cause of drug-induced retinopathy, and its exact mechanism still remains unclear. The incidence of retinal maculopathy induced by CQ has been reported to be about $10 \%$ - $25 \% 7$, and the incidence of retinopathy after more than 5 years of HCQ use is about $7.5 \%{ }^{8}$

Although HCQ is safer than CQ, both drugs can cause irreversible retinal damage, and their retinal toxicity mainly damages photoreceptor cells, mainly in the form of degeneration of the outer nuclear layer of the retina in the early stage, and patients are usually asymptomatic, but show abnormalities in fundus examination or diagnostic tests $^{9}$ and damage to the retinal pigment epithelium occurs in the late stage. ${ }^{10}$ If damage is found before RPE damage occurs and the drug is discontinued in a timely manner, central visual acuity can be preserved ${ }^{\mathrm{n}}$, and if found late, it can lead to irreversible visual loss, and there is no effective treatment. ${ }^{12}$ Therefore, in the treatment of COVID-19 patients with CQ and HCQ, it is necessary to guard against the risk of druginduced retinopathy, and it is recommended to perform relevant ophthalmic screening in a proper way to maintain the incidence of drug toxicity at a low level and make the drug application safer and more reliable. 
The mechanism by which CQ and HCQ produce retinal toxicity is still unclear, and current studies mostly focus on their effects on retinal cell metabolism and function. Evaluation of rhesus monkey eye tissues chronically administered CQ showed that CQ was widely bound in pigmented ocular tissues (RPE, iris, choroid, and ciliary body) and eventually accumulated in the retina, which may represent one of the causes for retinal toxicity. ${ }^{13}$ The study of RPE cells treated with CQ and HCQ found that both CQ and HCQ inhibited the uptake of organic anion-transporting polypeptide $1 \mathrm{~A} 2$ and inhibited the cyclic absorption of all-trans retinol in RPE cells, suggesting that they might have an effect on the visual cycle. ${ }^{14}$

It has been suggested in previous studies that melanin in RPE may concentrate HCQ and enhance its toxicity, but melanin also has a role in helping RPE cells to remove intrinsic toxic substances. ${ }^{15,16} \mathrm{An}$ in vitro study has also found that CQ and HCQ can increase the permeability of RPE cells and may also exert some effects on the function of RPE cells. ${ }^{17}$ A recent study found that the toxicity of CQ on RPE cells might be related to the downregulation of p15o glued protein expression, which in turn inhibited the proliferation and microtubule nucleation of RPE cells. ${ }^{18}$

Other scholars have proposed that HCQ-induced retinal degeneration occurs in the retinal photoreceptor cell layer; since HCQ can affect the $\mathrm{pH}$ and function of lysosomes, it may affect the autophagic function of RPE, which in turn affects the stability of photoreceptor cell membranes and ultimately produces retinal toxicity. ${ }^{10-19}$ In a study of optic ganglion cells exposed to CQ, it was found that the retinal toxicity of CQ might affect visual conduction by changing the dynamics of acid sensing ion channel a in RGCs. ${ }^{20}$ These studies, however, focus on the acute effects of drugs on retinal cells and do not fully reveal the exact mechanism of chronic retinal toxicity in clinical patients.

Dosage is the most important risk factor for retinal toxicity caused by CQ and HCQ. The safe dose of CQ and HCQ recommended by the American Academy of Ophthalmology in 2016 Recommendations on Screening for Chloroquine (CQ) and Hydroxychloroquine (HCQ)
Retinopathy is $2.3 \mathrm{mg} / \mathrm{kg}$ and $5 \mathrm{mg} / \mathrm{kg}$ daily, respectively, and it is pointed out that attention should still be paid to adjusting the dose for patients with short stature and low body mass. ${ }^{21}$

A previous study has found that the application of a dose $>5.0 \mathrm{mg} / \mathrm{kg}$ significantly increases the risk of retinopathy, especially the use of extremely high doses. ${ }^{8}$ Recent studies have revealed that the incidence of retinopathy within 1 to 2 years can be $25 \%$ to $40 \%$ in patients treated with HCQ at $800-$ $1000 \mathrm{mg}$ (approximately $20 \mathrm{mg} / \mathrm{kg}$ ) daily. ${ }^{22-23} \mathrm{In}$ contrast, the incidence of retinopathy within 5 years was less than $1 \%$ in case of HCQ treatment at low doses of $4.0-5.0 \mathrm{mg} / \mathrm{kg}$. ${ }^{8}$ Therefore, the use of CQ or HCQ in the treatment of COVID-19 should be controlled as far as possible within the safe dose range. At present, in the clinical studies registered in China for the treatment of COVID-19, the recommended dosage of chloroquine phosphate is $0.5 \sim 1 \mathrm{~g}$ oral daily. ${ }^{24}$ The duration of medication is inseparable from the dose of medication and is also a risk factor for retinal toxicity caused by CQ and HCQ. Patients with retinopathy caused by CQ and HCQ mostly have a long history of medication, but acute toxic retinopathy caused by high-dose chloroquine requires increased vigilance. Because $C Q$ and HCQ are slowly excreted from the human body and carry some risks after long-term use even at the recommended dose, a previous study has pointed out that the cumulative dose of the drug may be associated with the occurrence of retinopathy. ${ }^{25}$ Therefore, it is recommended that, for patients who use CQ and HCQ for a long time, their the previous medication history after starting treatment with CQ or HCQ should be carefully reviewed and attention should be paid to the presence or absence of visual impairment during their medication, and the patients should cooperate with timely screening.

CQ and HCQ are mainly eliminated via the kidneys, and a study has found that patients with renal diseases are at higher risk of drug toxicity. ${ }^{26}$ The liver is also involved in the metabolism of CQ and HCQ, but there is no clear evidence of an association between liver disease and retinal toxicity. Since critically ill patients with COVID-19 often present with systemic multiple organ failure $^{27}$, and unpredictable levels in blood may occur with impaired liver and kidney function, so the dose used and the frequency of ophthalmic 
screening are recommended to be adjusted moderately according to the patient's plasma concentration. The patients with retinal or macular disease may be at a higher risk for retinopathy, and it is clinically recommended that the drugs with retinal toxicity should not be used if patients have the underlying diseases of retinal dystrophy or severe degeneration. At the same time, patients with retinal or macular diseases may lead to abnormal testing results, which may interfere with ophthalmic screening during medication. Therefore, caution should be exercised for the use of CQ and HCQ in patients with underlying eye diseases. ${ }^{21}$

It is recommended to perform proper baseline examination to rule out retinal or macular diseases and learn the basic conditions of the patient's retina. Clinically, it is believed that elderly patients may be at risk of retinopathy due to their weak resistance to drug toxicity and slow rate of drug metabolism. However, recent demographic studies have found no definitive link between age and the risk of ocular toxicity with drugs. ${ }^{8}$ It has been shown that some patients with $\mathrm{ABCA}_{4}$ gene abnormalities have genetic predisposition to HCQ retinal toxicity ${ }^{28} \mathrm{~A}$ new study has suggested that some non-pathogenic polymorphic $\mathrm{ABCA}_{4}$, such as small alleles of common genetic variants in $\mathrm{ABCA}_{4}$, may instead have a protective effect. ${ }^{29}$ Genetic polymorphisms in cytochrome $\mathrm{P} 450$ may also affect plasma concentrations as well as the ocular toxicity of drugs..$^{30}$

Early patients with retinopathy caused by CQ and HCQ usually present with normal vision or even without any visual symptoms, and only a few patients may present with paracentral scotomas and fine punctate pigment spots in the macular area during reading. If exposure to drugs is continued, the dysfunctional area expands, RPE is involved, characteristic bullseye maculopathy may occur in the fundus, visual acuity decreases, and late lesions invade the fovea, resulting in loss of visual acuity; in addition, macular cystic edema may also occur, with extensive RPE and retinal atrophy. ${ }^{31.32}$ Its bullseye like typical manifestations occur due to RPE depigmentation in a circle of the macular central fovea. European patients mostly present with typical parafoveal photoreceptor layer injury33, while Asian patients often present with injury near the vascular arch at the parafoveal margin. ${ }^{34}$ Some auxiliary examinations show abnormal results, such as multifocal electroretinography showing diminished perifoveal response and enhanced fundus autofluorescence, spectral-domain optical coherence tomography showing parafoveal thinning and destruction of the hierarchy of the external membrane and ellipsoid band, while the structure just below the fovea being relatively intact, presenting a flying saucer sign. ${ }^{35}$ Visual field detection results are characterized by bilateral reproducible persistent paracentral or central visual field blind spots under stimulation on the white light threshold and may also have other visual field abnormalities. ${ }^{36}$ If retinopathy occurs relatively slowly after medication, retinopathy should be closely monitored. If it is not enough to affect vision, it is unnecessary to discontinue the drug. Once retinal toxicity is significantly aggravated, the risk of continuing medication should be discussed with an ophthalmologist. In addition, the use of CQ and HCQ may cause vortex keratopathy or whorl keratopathy, but it is mostly reversible after discontinuation of the drug and there is no direct evidence related to the severity of retinopathy. 37,38

Retinal damage caused by HCQ and CQ is often irreversible, and screening, although not reducing drug-induced retinal damage, can detect retinal toxicity before vision is significantly affected, so as to guide medication. HCQ and CQ are potentially effective drugs for the treatment of COVID-19, and screening should be regarded as a means of preventing severe retinal damage to support the safe application of HCQ or CQ in patients and detect the potential risk of retinal damage at an early stage. If a patient has retinopathy, such as maculopathy, glaucoma, and so on, the drug should be used with caution, or the lower dose should be maintained. If there are signs of retinal damage during treatment, ophthalmic examinations should be repeated to verify the examination results. If it is determined that the damage occurs, the dose should be adjusted according to the patient's actual conditions to control the progression of damage. For screening retinopathy due to early HCQ and CQ, visual field testing has good sensitivity, but reliability cannot be guaranteed due to the presence of subjective factors in patients. Some objective tests show a good sensitivity for early lesions, such as mfERG, SD-OCT and FAF. Therefore, it is recommended to combine with objective examination at the 
same time of visual field testing to produce reliable screening results.

Under the condition of excluding patient's subjective factors, perimetry is a more sensitive method to detect the retinal condition of patients. For the screening of retinopathy caused by HCQ and CQ, foreign studies mostly recommend the use of the standard procedure of 10-2 visual fields with a white target, and it is believed that the test results have a good sensitivity. ${ }^{21}$ At the same time, the subjectivity of perimetry cannot be neglected, and the results of perimetry are not enough to be used as a confirmation of retinal damage, and repeated testing should be performed in combination with other objective test results. With the development of injuries caused by $C Q$ and HCQ, SD-OCT examination can find damage to the parafoveal photoreceptor cell layer in European patients, while damage near the extrafoveal vascular arch is more common in Asian patients. Due to the different location of retinopathy in Asian patients compared to European patients, it is particularly important to perform wide-angle scanning or direct scanning of the vascular arch region in Asian patients. SDOCT examination in patients with advanced retinopathy caused by HCQ may present with a typical flying saucer sign, that is, a relatively normal foveal retina, thinning of the outer retina of the parafovea, destruction of structures such as the external membrane and ellipsoid zone, resembling a flying saucer shape. 35

SD-OCT may not be as sensitive as visual field testing and mfERG, but when local retinal thinning occurs, it is basically certain that retinal damage has been caused. mfERG can objectively record early lesions in the para-retinal fovea and extramacular regions by electrophysiological means, with a sensitivity close to that of perimetry, which can provide objective evidence for patients with abnormal perimetry. ${ }^{39}$ Studies have found that mfERG signal may be weakened after HCQ administration ${ }^{40}$, and mfERG signal may be recovered to some extent after drug discontinuation and has a negative correlation with drug accumulation ${ }^{41}$, suggesting that mfERG can be used as a reference index for drug dose adjustment. However, due to the low application rate of mfERG, there are limited physicians who can skillfully complete the examination procedure, which limits the application of this method to some extent. FAF is of high value for the examination of posterior fundus injury and is most sensitive for the examination of RPE damage, which may detect early injury in macular foveal or extramacular photoreceptor before SD-OCT shows retinal thinning. ${ }^{42}$ When the damage reaches a late stage, RPE loss is manifested as a dark area with reduced autofluorescence. Wide-angle image mode should be used for Chinese patients when CQ and HCQ retinal toxicity is confirmed by FAF testing. When ophthalmoscopy can detect visible changes in the fundus, retinopathy often has reached an advanced stage of irreversible damage, and retinopathy can be detected earlier by other techniques. ${ }^{43}$

At the same time, ophthalmoscopy performed at a close doctor-patient distance will increase the risk of exposure of ophthalmologists, so ophthalmoscopy is not recommended. Tests such as time-domain optical coherence tomography, fundus fluorescein angiography, full-field electroretinography, Amsler chart, color vision test, and electrooculography are not recommended screening methods due to insufficient sensitivity. ${ }^{23,44}$ It is worth mentioning that although full-field electroretinography cannot detect early retinopathy, it may be helpful in judging damage other than the macula. ${ }^{45}$

There is no specific treatment for retinopathy caused by CQ and HCQ, so early preventive screening appears to be more important. Early macular changes may be reversible after discontinuation of the drug and damages caused at the advanced stage are irreversible. For most patients, retinopathy may tend to be stable after drug discontinuation, but some patients still have the possibility of progression of lesions after drug discontinuation, which may be related to slower metabolism of HQ. ${ }^{27}$ Once the occurrence of retinopathy is confirmed, clinicians should consider prompt discontinuation of medication and inform patients of the risk of further visual impairment based on the severity of retinopathy. It is generally recommended that patients with retinopathy caused by CQ and HCQ avoid excessive exposure to sunlight and ingest sufficient lutein and zeaxanthin to protect 
photoreceptor cells in the fovea; however, the practical value of these recommendations is unclear due to the lack of clinical evidence. ${ }^{21}$

\section{CONCLUSION}

Chloroquine and hydroxychloroquine have definite inhibitory effects on SARS-CoV-2, and they are recommended for the treatment of COVID-19. Retinal damage caused by it occurs mostly after long-term medication; however, due to different physical constitutions in different patients, there are also some differences in tolerance to the drug, short-term application of high-dose drugs can significantly increase the risk of retinal damage. Therefore, in the treatment of COVID-19 with CQ and HCQ, it is important to recognize the characteristics of retinopathy caused by such drugs, pay attention to adjusting the dose according to the actual conditions of patients, use necessary ophthalmic screening for patients at higher risk, and use relative caution in patients with retinal or macular diseases. The application of risk screening methods can help detect early retinal damage and guide clinical adjustment of medication dose. For patients with retinopathy, if necessary, the drug should be discontinued and switched to other therapeutic drugs. At present, CQ and HCQ have achieved promising therapeutic effects in the treatment of COVID-19. The proportion and severity of toxic retinopathy during the treatment remain to be further studied. Before the exact research results are obtained, we still need to exercise caution during the application of CQ and HCQ.

\section{REFERENCES}

1. Wang M, Cao R, Zhang L et al. Remdesivir and chloroquine effectively inhibit the recently emerged novel coronavirus $(2019-\mathrm{nCoV})$ in vitro. Cell Res. 2020;30(3):269-71.

2. Jorge A, Ung C, Young LH et al. Hydroxychloroquine retinopathy-implications of research advances for rheumatology care. Nat Rev Rheumatol 2018;14(12):693-703.

3. Carlos WG, Dela Cruz CZ, Cao B et al. Novel Wuhan (2019-nCoV) Coronavirus. Am J Respiratory Crit Med. 2020;201 (4): 7-8.

4. Lan J, Ge J, Yu J et al. Structure of the SARS$\mathrm{CoV}-2$ spike receptor binding domain bound to the ACE2 receptor. Nature 2020;581 (7807):215-20.
5. Zhou P, Yang XL, Wang XG, et al. A pneumonia outbreak associated with new coronavirus of probablw bat origin. Nature 2020; 579(7798):270-3. 6.Xu X, Chen P, Wang, J et al. Evolution of the novel coronavirus from the ongoing Wuhan outbreak and modelling of its spike protein for risk of human transmission. Sci China Life Sci. 2020;63(3):457-6o.

7. Finbloom DS, Silver K, Newsome DA, et al. Comparison of hydroxychloroquine and chloroquine use and the development of retinal toxicity. J Rheumatol. 1985;12(4):692-4.

8. Melles RB, Marmor MF. The risk of toxic retinopathy in patients on long term hydroxychloroquine therapy. JAMA Ophthalmol. 2014;132(12):1453-6o.

9. Nika M, Blachley TS, Edwards P et al. Regular examinations for toxic maculopathy in long term chloroquine or hydroxychloroquine users. JAMA Ophthalmol. 2014; 132(10):1199-208.

10. Marmor MF. Comparison of screening procedure in hydroxychloroquine toxicity. Arch Ophthalmol. 2012;130(4):461-9.

11. Marmor MF, Hu J. Effect of disease stage on progression of hydroxychloroquine retinopathy. JAMA Ophthalmol. 2014;132(9):1105-12.

12. Marmor MF, Melles RB. Hydroxychloroquine and the retina. JAMA Ophthalmol. 2015;313(8):8478.

13. Rosenthal AR, Kolb H, Bergsma D, et al. Chloroquine retinopathy in the rhesus monkey. Invest Ophthalmol Vis Sci. 1978;17(12):1158-75.

14. Xu C, Zhu L, Chan T, et al. Chloroquine and hydroxychloroquine are novel inhibitors of human organic anion transporting polypeptide $1 \mathrm{~A} 2$. J Pharm Sci. 2016;105(2):884-90.

15. Lee MG, Kim SJ, Ham DI, et al. Macular retinal ganglion cell inner plexiform layer thickness in patients on hydroxychloroquine therapy. Invest Ophthalmol Vis Sci. 2014;56(1): 396-402.

16.deSisterness $\mathrm{L}$, $\mathrm{Hu}$ J, Rubin $\mathrm{DL}$, et al. Localization of damage in progressive hydroxychloroquine retinopathy on and off the drug: inner versus outer retina, parafovea versus peripheral fovea. Invest Ophthalmol Vis Sci. 2015; 56(5)3415-26.

17. Korthagen NM, Bastiaans J, vanMeurs JC, et al. Chloroquine and hydroxychloroquine increase retinal pigment epithelial layer permeability. J Biochem Mol Toxicol. 2015;29(7):299-304. 
18. Chen TY, Lien WC, Cheng HL, Kuan TS, Sheu SY, Wang CY. Chloroquine inhibits human retina pigmented epithelial cell growth and microtubule nucleation by downregulating pi5oglued. J Cell Physiol.

2019;234(7):10445-7.

https://doi.org/10.1002/jcp.27712.

19. Yusuf IH, Sharma S, Luqmani R, Downes SM. Hydroxychloroquine retinopathy. Eye (Lond). 2017;31(6):828-45.

https://doi.org/10.1038/eye.2016.298.

20. Li X, Fei J, Lei Z, et al. Chloroquine impairs visual transduction via modulation of acid sensing ion channel 1a. Toxicol Lett. 2014;228(3):200-6.

21. Marmor MF, Kellner U, Lai TY, Melles RB, Mieler WF. American Academy of Ophthalmology. Recommendations on Screening for Chloroquine and Hydroxychloroquine Retinopathy (2016 Revision). Ophthalmology. 2016;123(6):1386-94.

22. Leung LS, Neal JW, Wakelee HA, Sequist LV, Marmor MF. Rapid Onset of Retinal Toxicity From High-Dose Hydroxychloroquine Given for Cancer Therapy. Am J Ophthalmol. 2015;16o(4):799-805.e1. https://doi.org/10.1016/j.ajo.2015.07.012.

23. Navajas EV, Krema H, Hammoudi DS, Lipton JH, Simpson ER, Boyd S, Easterbrook M. Retinal toxicity of high-dose hydroxychloroquine in patients with chronic graft-versus-host disease. Can J Ophthalmol. 2015;50(6):442-50.

24. Gao J, Tian Z, Yang X. Breakthrough: Chloroquine phosphate has shown apparent efficacy in treatment of COVID-19 associated pneumonia in clinical studies. Biosci Trends. 2020 16;14(1):72-73.

25. Marmor MF, Kellner U, Lai TY, Lyons JS, Mieler WF. American Academy of Ophthalmology. Revised recommendations on screening for chloroquine and hydroxychloroquine retinopathy. Ophthalmology. 2011;118(2):415-22.

26. Chiang E, Jampol LM, Fawzi AA. Retinal toxicity found in a patient with systemic lupus erythematosus prior to 5 years of treatment with hydroxychloroquine. Rheumatology (Oxford). 2014;53(11):2001.

https://doi.org/10.1093/rheumatology/keu317.

27. Chen N, Zhou M, Dong X, Qu J, Gong F, Han Y, et al. Epidemiological and clinical characteristics of 99 cases of 2019 novel coronavirus pneumonia in Wuhan, China: a descriptive study. Lancet. 2020;395(10223):507-13.

28. Shroyer NF, Lewis RA, Lupski JR. Analysis of the $\mathrm{ABCR}\left(\mathrm{ABCA}_{4}\right)$ gene in 4-aminoquinoline retinopathy: is retinal toxicity by chloroquine and hydroxychloroquine related to Stargardt disease? Am J Ophthalmol. 2001;131(6):761-6.

29. Grassmann F, Bergholz R, Mändl J, Jägle $H$, Ruether K, Weber BH. Common synonymous variants in $\mathrm{ABCA}_{4}$ are protective for chloroquine induced maculopathy (toxic maculopathy). BMC Ophthalmol. 2015;15:18.

30. Lee JY, Vinayagamoorthy N, Han K, Kwok SK, Ju JH, Park KS, et al. Association of Polymorphisms of Cytochrome $\mathrm{P} 450$ 2D6 With Blood Hydroxychloroquine Levels in Patients With Systemic Lupus Erythematosus. Arthritis Rheumatol. 2016;68(1):184-90.

31. Modi YS, Singh RP. Bull's-Eye Maculopathy Associated with Hydroxychloroquine. N Engl J Med. 2019;380(17):1656.

32. Kellner S, Weinitz S, Farmand G, Kellner U. Cystoid macular oedema and epiretinal membrane formation during progression of chloroquine retinopathy after drug cessation. Br J Ophthalmol. 2014;98(2):200-6.

33. Melles RB, Marmor MF. Pericentral retinopathy and racial differences in hydroxychloroquine toxicity. Ophthalmology. 2015;122(1):110-6.

34. Lee DH, Melles RB, Joe SG, Lee JY, Kim JG, Lee CK, Yoo B, Koo BS, Kim JT, Marmor MF, Yoon YH. Pericentral hydroxychloroquine retinopathy in Korean patients. Ophthalmology. 2015;122(6):12526.

35. Chen E, Brown DM, Benz MS, Fish RH, Wong TP, Kim RY, et al. Spectral domain optical coherence tomography as an effective screening test for hydroxychloroquine retinopathy (the "flying saucer" sign). Clin Ophthalmol. 2010;4:11518.

36. Easterbrook M. An ophthalmological view on the efficacy and safety of chloroquine versus hydroxychloroquine. J Rheumatol. 1999;26(9):1866-8.

37. Paim-Marques L, Carneiro P, Verçosa IC, Appenzeller S. Corneal vortex keratopathy in childhood-onset systemic lupus erythematosus (cSLE). Clin Rheumatol. 2019;38(10):2851-5.

38. Lacava AC. Ocular complications of chloroquine and derivatives therapy. Arq Bras Oftalmol. 2010;73(4):384-9.

39. Lai TY, Ngai JW, Chan WM, Lam DS. Visual field and multifocal electroretinography and their correlations in patients on hydroxychloroquine therapy. Doc Ophthalmol. 2006 May;112(3):177-87. 4o. Maturi RK, Yu M, Weleber RG. Multifocal electroretinographic evaluation of long-term 
hydroxychloroquine users. Arch Ophthalmol. 2004;122(7):973-81.

41. Lyons JS, Severns ML. Using multifocal ERG ring ratios to detect and follow Plaquenil retinal toxicity: a review : Review of mfERG ring ratios in Plaquenil toxicity. Doc Ophthalmol. 2009;118(1):29-36.

42. Kellner U, Renner AB, Tillack H. Fundus autofluorescence and mfERG for early detection of retinal alterations in patients using chloroquine/hydroxychloroquine. Invest Ophthalmol Vis Sci. 2006;47(8):3531-8.

43. Missner S, Kellner U. Comparison of different screening methods for chloroquine/hydroxychloroquine retinopathy: multifocal electroretinography, color vision, perimetry, ophthalmoscopy, and fluorescein angiography. Graefes Arch Clin Exp Ophthalmol. 2012;250(3):319-25.

44. Eldeeb M, Chan EW, Omar A. Case Report: Hydroxychloroquine Retinopathy. Optom Vis Sci. 2018;95(6):545-9.

45. Nair AA, Marmor MF. ERG and other discriminators between advanced hydroxychloroquine retinopathy and retinitis pigmentosa. Doc Ophthalmol. 2017 Jun;134(3):17583.

Source of support: Nil, Conflict of interest: None declared

Bharti M. COVID-19 and Retinal Toxicity of Anti-Malarials. Int Healthc Res J. 2020;4(8):RV5RV11. https://doi.org/10.26440/IHRJ/0408.11364

\section{AUTHOR AFFILIATION:}

Masters in Pharmacy (Pharmacology), MDU, Rohtak 